\title{
Prophylactic Inhalation of L-Alanyl-L-Glutamine Enhances Heat Shock Protein 72 and Attenuates Endotoxin-Induced Lung Injury in Rats
}

\author{
I.-C. CHUANG ${ }^{1,2,3}$, M.-S. HUANG ${ }^{2,3}$, L.-J. HUANG ${ }^{4}$, S.-H. CHOU ${ }^{1,2}$, T.-N. TSAI ${ }^{2}$, \\ Y.-C. $\mathrm{CHEN}^{3}$, R.-C. YANG ${ }^{2,5}$ \\ ${ }^{1}$ Department of Respiratory Therapy, Kaohsiung Medical University, Kaohsiung, Taiwan, \\ ${ }^{2}$ Graduate Institute of Medicine, Kaohsiung Medical University, Kaohsiung, Taiwan, ${ }^{3}$ Division \\ of Respiratory and Critical Care Medicine, Department of Internal Medicine, Kaohsiung Medical \\ University Hospital, Kaohsiung, Taiwan, ${ }^{4}$ Center of Teaching and Research, Kaohsiung Municipal \\ Ta-Tung Hospital, Kaohsiung Medical University Hospital, Kaohsiung, Taiwan, ${ }^{5}$ Department \\ of Pediatrics, Changhua Christian Hospital, Changhua, Taiwan
}

Received May 14, 2014

Accepted September 26, 2014

On-line December 3, 2014

\section{Summary}

Studies have demonstrated that heat shock protein 70 (HSP70) plays an important role in the protection of stressed organisms. The development of strategies for enhancing HSPs expression may provide novel means of minimizing inflammatory lung conditions, such as acute lung injury. This study aimed to examine the effect of L-alanyl-L-glutamine (GLN) inhalation in enhancing pulmonary HSP72 (inducible HSP70) expression and attenuating lung damage in a model of acute lung injury induced by Lipopolysaccharide (LPS) inhalation. The experimental rats were randomly assigned to one of four experimental groups: (1) NS: saline inhalation; (2) NS-LPS: pretreatment by saline inhalation $12 \mathrm{~h}$ before LPS inhalation; (3) GLN: glutamine inhalation; (4) GLN-LPS: pretreatment by glutamine inhalation $12 \mathrm{~h}$ before LPS inhalation. The results show that GLN compared with saline administration, led to significant increase in lung HSP72 both in non LPS-treated rats and LPS-treated rats. In LPStreated rats, pretreatment by GLN inhalation produced less lung injury as evidenced by the decrease in lung injury score and dramatic decrease in lactate dehydrogenase (LDH) activity and polymorphonuclear leukocyte cell differentiation counts (PMN \%) in the bronchoalveolar lavage fluid. The study indicates that prophylactic glutamine inhalation associated with the enhancement of HSP72 synthesis attenuates tissue damage in experimental lung injury.

\section{Key words}

Glutamine • Heat shock protein 72 • Acute lung injury • Aerosol therapy

\section{Corresponding author}

R.-C. Yang, Graduate Institute of Medicine, Kaohsiung Medical University \#100, Shih-Chuan 1st Rd., Kaohsiung Country, Taiwan, 807, ROC. Fax: 886-7-3234687. E-mail: rechya@kmu.edu.tw

\section{Introduction}

A self-protective phenomenon, a so-called heat shock response (HSR) was first observed in Drosophila embryos by Ritossa (1962). Sudden heat stress can induce the expression of a set of proteins in living cells referred to as stress or heat shock proteins (HSPs). They are synthesized constitutively or inducibly under a wide variety of stresses. Among the $70-\mathrm{kDa}$ family of HSP (HSP70s), inducible HSP70, also named as HSP72, is the most important protein in the HSP family playing a vital role in cellular protection against injuries in the presence of various stresses (Dong et al. 2005, De Maio 1999, Karinch et al. 2001, Schmitt et al. 2007). Several reports have demonstrated that external heating or administration of sodium arsenite results in expression of HSP72 and 
significantly reduces end-organ injury and mortality rate resulting from endotoxemia caused by Escherichia coli lipopolysaccharide (LPS) or cecal ligation and puncture (CLP) (Bruemmer et al. 2001, Hotchkiss et al. 1993, Villa et al. 1994, Ribeiro et al. 1994, Chen et al. 2003, Huang et al. 2012). However, neither heat stress nor sodium arsenite are clinically relevant. The development of practical strategies for enhancing HSP72 expression may provide novel means of therapeutic intervention in critically ill patients.

Glutamine (GLN), a nonessential amino acid, is the most abundant in the healthy human being and has also been considered a "conditionally essential" amino acid in states of serious illness or injury (Roth 2008, Bongers et al. 2007). More recently, studies have supported the evidence that GLN mediated enhancement of HSP72 works against inflammatory injury and illness in experimental settings (Morrison et al. 2006, Singleton et al. 2005a,b). Studies have implied that septic shock and acute lung injury lead to a down regulation of HSP70 expression in the lung. The decrease in HSP70 may lead to a worsening of lung tissue metabolism, subsequent lung injury, and organ failure. This decreased HSP70 expression can also be corrected by administration of GLN (Wischmeyer et al. 2001, Singleton and Wischmeyer 2007). Research in animal models of sepsis and lung injury demonstrates the beneficial effect of supplemental GLN on survival, metabolic function, immune function and tissue protection (Zhang et al. 2009, Jing et al. 2007, Singleton et al. 2005a,b). Clinical trials in human subjects have also demonstrated that GLN reduces infectious complications, inflammatory response and ICU mortality (Vermeulen et al. 2007, Wischmeyer 2008, Sufit et al. 2012). Now, increasing evidence suggests GLN as a nontoxic, clinically relevant enhancer of HSP72 expression in critically ill patients. GLN appears to be a possible candidate for novel cytoprotective drugs by enhancement of HSP72 expression. Moreover, reported routes of administration for exogenous glutamine to prevent glutamine-deficiency in critically ill patients are mainly oral, parenteral, enteral and intravenous. Therefore, we designed a practical method to administer GLN via airway inhalation. Inhalation therapy refers to the delivery of drugs directly to the lungs with minimal risks of systemic effects (Rubin 2010). Besides, the onset of action for nebulized GLN is substantially faster than for oral or parenteral formulation. Apart from the route of administration, other points of interest are the expression of HSP72 evoked by a single dose of GLN inhalation in the lung tissue and its beneficial effects. This study aimed to examine the hypothesis that inhalation of nebulized GLN could enhance pulmonary HSP72 expression and prophylactically attenuate lung damage in a rat model of acute lung injury induced by lipopolysaccharide (LPS) inhalation.

\section{Materials and Methods}

Animals

Experiments were performed on adult male Sprague-Dawley (SD) rats weighing 280-380 g. Room temperature was maintained at $21 \pm 1^{\circ} \mathrm{C}$ under a $12 \mathrm{~h}$ light-dark regimen. Food and water were provided ad libitum. Our study adhered to the guidelines of the National Institutes of Health for the use of experimental animals and all experiments were approved by the Committee for the Use of Experimental Animals of Kaohsiung Medical University (IACUC Approval NO: 99062).

Time course effect of GLN inhalation on HSP72 expression

The time-response curve from 0 to $20 \mathrm{~h}(0 \mathrm{~h}, 4 \mathrm{~h}$, $8 \mathrm{~h}, 12 \mathrm{~h}, 16 \mathrm{~h}, 20 \mathrm{~h}$ ) after GLN inhalation was examined at a dose of $1.5 \mathrm{~g}$ GLN. Lung tissues were harvested at various time points after GLN inhalation. HSP72 expressions were analyzed by western blot and the 12-h time point was chosen to obtain the maximal response of HSP72 protein expression.

\section{Experimental protocol}

Rats were randomly assigned to one of four experimental groups, (1) NS $(\mathrm{n}=8)$ : saline placebo inhalation; (2) NS-LPS $(\mathrm{n}=8)$ : pretreatment by saline inhalation $12 \mathrm{~h}$ before LPS inhalation; (3) GLN ( $\mathrm{n}=8$ ): glutamine inhalation; (4) GLN-LPS ( $\mathrm{n}=8)$ : pretreatment by glutamine inhalation $12 \mathrm{~h}$ before LPS inhalation. For all manipulations, rats were given intraperitoneal injections of ketamine $(80 \mathrm{mg} / \mathrm{kg})$ and xylazine $(12 \mathrm{mg} / \mathrm{kg})$. At the end of the experiment, the rats were sacrificed and samples were harvested under a lethal dose of ketamine $(100 \mathrm{mg} / \mathrm{kg})$. The NS and GLN groups were devitalized at $12 \mathrm{~h}$ after inhalation treatment whereas rats from NS-LPS and GLN-LPS groups receiving NS or GLN prophylactic inhalation respectively were sacrificed at $8 \mathrm{~h}$ after LPS inhalation. The degree of lung injury was assessed by various parameters including pathologic 
change in lung tissue, and lactate dehydrogenase (LDH) activity and polymorphonuclear leukocyte cell differentiation counts (PMN \%) in the bronchoalveolar lavage fluid (BALF). The HSP72 of lung tissue was examined by Western blot analysis.

\section{Drug preparation and delivery}

Lipopolysaccharides and L-alanyl-L-glutamine were purchased from Sigma Chemical (St. Louis, MO, USA). GLN was prepared as a $38 \%$ solution dissolved in $0.9 \%$ saline solution. Approximately four milliliters of GLN solution were administered via nebulization to yield a single dose of $1.5 \mathrm{~g}$ GLN. The LPS stock aliquot $(2 \mathrm{mg} / \mathrm{ml})$ was reconstituted by dissolving Escherichiacoli-derived LPS in endotoxin free sterile $0.9 \%$ saline. The LPS solution was prepared by diluting $1 \mathrm{ml}$ LPS stock in $1 \mathrm{ml} 0.9 \%$ saline for nebulization. Saline solution was used as a placebo. Nebulization of inhaled agents was performed by Aeroneb Pro (Aerogen) and delivered via a nose cone (Rodent anesthesia mask, Braintree Scientific, Inc.). The treatment time was approximately 15-20 min for GLN and 5-10 min for LPS.

\section{BALF analysis}

The left lung was intratracheally lavaged twice with $0.9 \%$ saline $(2.5 \mathrm{ml}$ per lavage). The effluents were centrifuged at $3,000 \mathrm{rpm}$ at $4{ }^{\circ} \mathrm{C}$ for $10 \mathrm{~min}$. The BALF supernatant was immediately examined for LDH activity. $\mathrm{PMN} \%$ was used to measure migration of PMN into the alveoli. To perform cell differentials, cells were fixed on glass slides by using cytospin and were stained with Wright-Geimsa. The ratio of PMN to total cell (PMN \%) was counted to be at least 200 cells/smear (Ong et al. 2003).

\section{HSP72 detection in lung tissue}

Western blotting was performed as previously described (Wang et al. 2012). The monoclonal antiHSP72 antibody (Enzo Life Science, New York, USA) were utilized as the primary antibody. The anti-mouse immunoglobulin G (GE Healthcare) conjugated with peroxidase was used as the secondary antibody. Densitometric analysis was performed using Bio-1D V.97 software (Vilber Lourmat, Germany).

\section{Lung pathology}

Histology was assessed by hematoxylin and eosin staining. For quantification of lung injury score, various degrees of lung injury score were assessed as degree 0 to 4 in two categories: edema and cellular infiltration. A score of 0 represented normal findings and scores of $1,2,3$ and 4 represented mild $(<25 \%)$, moderate $(25-50 \%)$, severe $(50-75 \%)$ and very severe $(>75 \%)$ lung involvement. The histopathological assessment was performed in a blind fashion by several laboratory assistants. Each one gave a score for edema and cell infiltration from 0 to 4 . The individual scores were added together to obtain a final score, ranging from 0-8 (Chuang et al. 2007, Koga et al. 2010).

\section{Statistical analysis}

Values are expressed as means \pm standard deviation (SD). Statistical evaluation of the group comparison was made with one-way ANOVA and paired Student $\mathrm{t}$ test. Differences were considered statistically significant at $P<0.05$.

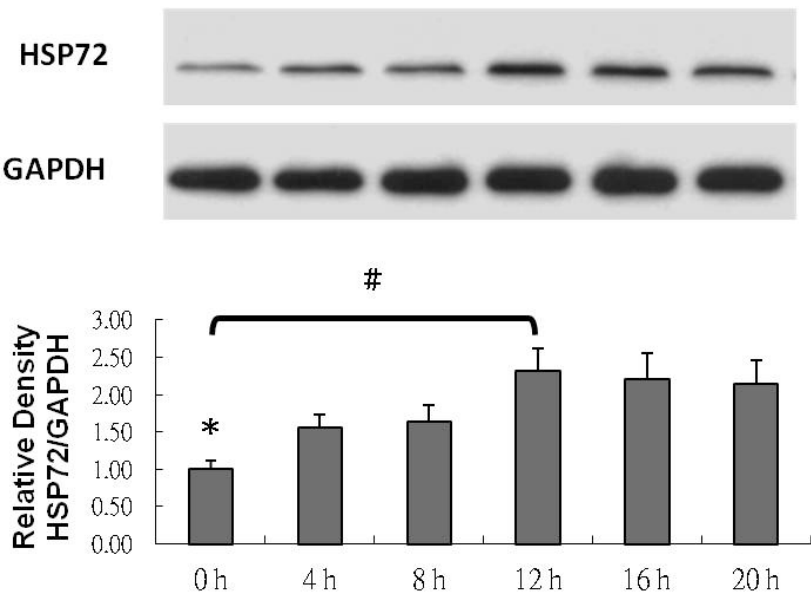

Fig. 1. Time course effect of inhaled GLN on HSP72 expression in lung tissue of rats. HSP72 expression was analyzed by Western blotting analysis. Relative density refers to the ratio of HSP72 to GAPDH. Image represents results found in triplicate. Relative densitometric results are expressed as mean $\pm S D$ of levels relative to that of the control group $(0 \mathrm{~h}), * \mathrm{P}<0.05 \mathrm{vs}$. all other groups. Notable findings include a peaking at $12 \mathrm{~h}\left({ }^{\#} \mathrm{P}<0.0001\right.$ vs. $0 \mathrm{~h}$ ) and a non-significant decay after $16 \mathrm{~h}$ and $20 \mathrm{~h}$ (compared with $12 \mathrm{~h}, \mathrm{P}>0.5, \mathrm{P}>0.3$ ).

\section{Results}

Time course effect of GLN inhalation on HSP72 expression

Figure 1 shows the time-response graph for experiments examining multiple time points from 0 to $20 \mathrm{~h}$ at a dose of $1.5 \mathrm{~g}$ GLN inhalation for HSP72 induction. There was a significant variation in HSP72 expression in all GLN treated animals at different time points (compared with $0 \mathrm{~h}, \mathrm{P}<0.05$ ). Notable findings 
include a peaking at $12 \mathrm{~h}$ (compared with $0 \mathrm{~h}, \mathrm{P}<0.0001$ ) and a non-significant decay after $16 \mathrm{~h}$ and $20 \mathrm{~h}$ (compared with $12 \mathrm{~h}, \mathrm{P}>0.5, \mathrm{P}>0.3$ ).

GLN enhances HSP72 expression in non LPS-treated and LPS-treated rats

As determined by western blot analysis (Fig. 2), glutamine compared with saline inhalation led to a significant increase in lung HSP72 both in non LPS-treated rats (NS compared with GLN, $1 \pm 0.15$ vs. 2.10 $\pm 0.46, \quad \mathrm{P}<0.0001$ ) and LPS-treated rats (NS-LPS compared with GLN-LPS, $0.68 \pm 0.16$ vs. $1.33 \pm 0.27$, $\mathrm{P}<0.001)$. LPS inhalation down-regulated HSP72 expression compared with saline inhaled animals (NS vs. NS-LPS, $\mathrm{P}<0.05$ ).

\section{GLN's protective effects on LPS induced lung injury}

To examine the effect of GLN inhalation on protection from LPS induced lung injury, a single dose of $1.5 \mathrm{~g} \mathrm{GLN}$ or saline solution was used to pretreat the subject rats $12 \mathrm{~h}$ before LPS inhalation. In LPS-treated rats, pretreatment by glutamine inhalation (GLN-LPS) significantly increased lung HSP72 expression compared with the saline pretreatment group (NS-LPS) (Fig. 2, $\mathrm{P}<0.001)$. The degree of lung injury was assessed by various parameters including pathologic change in lung tissue, and LDH activity and differential PMN \% in the BALF.

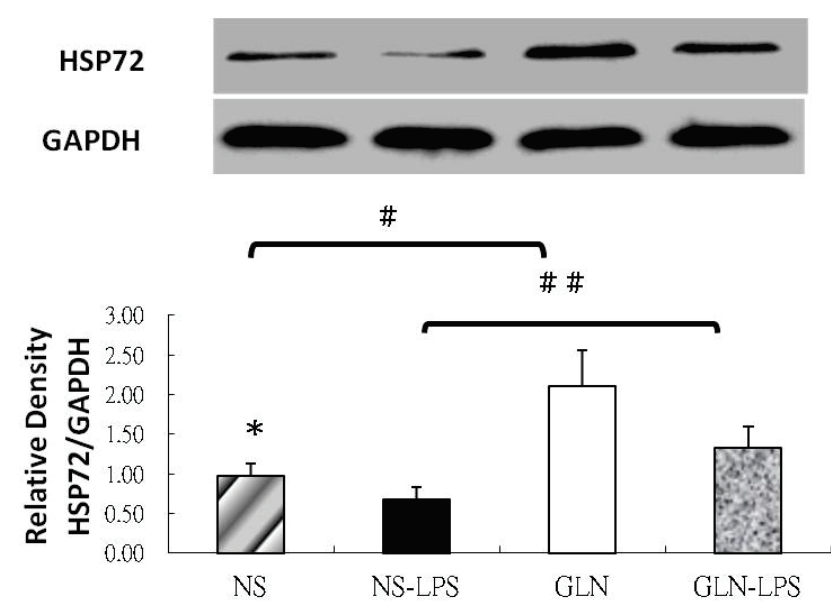

Fig. 2. Western blot analysis of HSP72 in lung tissue from different groups of rats. HSP72 expression was analyzed by Western blot analysis. Relative density refers to the ratio of HSP72 to GAPDH. Image represents results found in triplicate. Relative densitometric results are expressed as mean \pm SD of levels relative to that of the control group (NS), $* P<0.05 \mathrm{vs}$. all other groups. LPS inhalation down-regulates HSP72 expression. Inhaled GLN up-regulates lung HSP72 expression both in non LPS-treated rats ( ${ }^{\#} \mathrm{P}<0.0001 \mathrm{NS}$ vs. GLN) and LPS-treated rats ( \#\# $\mathrm{P}<0.001$ NS-LPS vs. GLN-LPS).
Lung pathology

Inhaled LPS elicited marked pulmonary edema and infiltration of PMN into bronchoalveolar space (Fig. 3A). The histomicrograph and quantification of lung injury score revealed that GLN pretreatment significantly attenuated the extent of lung injury (NS-LPS compared with GLN-LPS, $6.5 \pm 1.4$ vs. 3.9 $\pm 0.9, \mathrm{P}<0.0001$, Fig. 3B). Animals receiving GLN only did not show pathological changes in lung tissue.

A.
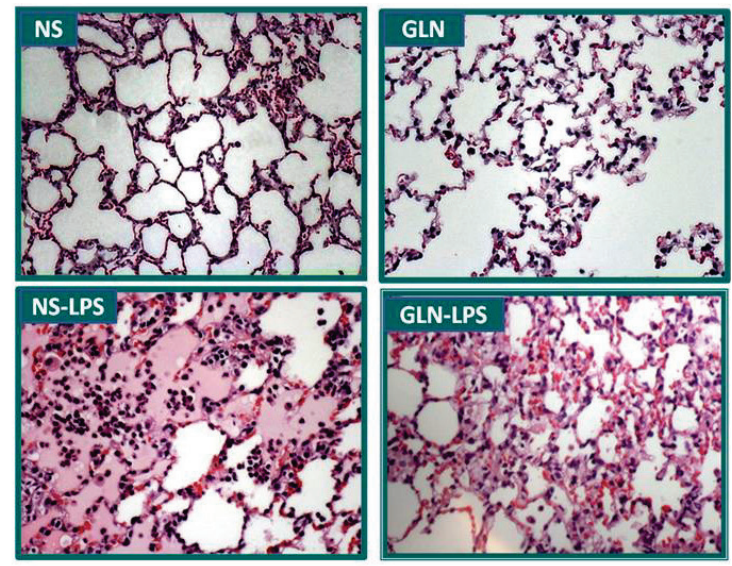

B.

\#

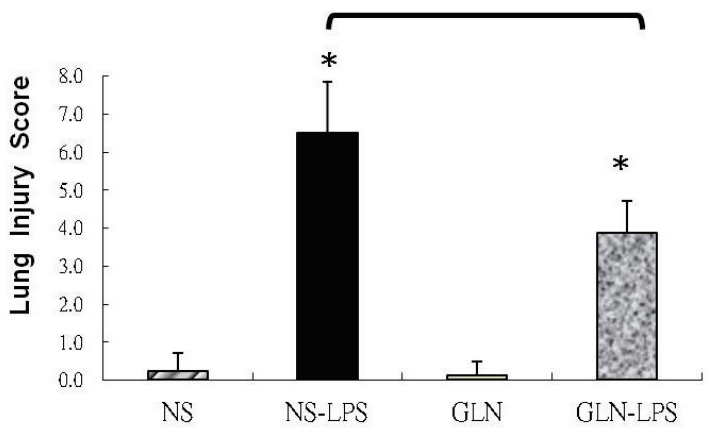

Fig. 3. A. Illustrative microphotographs showing the lung histology of lung tissue obtained in four experimental groups. Note the severe pulmonary edema and cellular infiltration in the NS-LPS group and the attenuation of lung pathology with GLN pretreatment (GLN-LPS). Hematoxylin and eosin stain, original magnification $\times 20$. B. The data were obtained from scoring the lung pathological changes (edema formation and inflammatory cells infiltration) in rat lungs. ${ }^{*} \mathrm{P}<0.0001$ vs. NS, ${ }^{*} \mathrm{P}<0.0001$ NSLPS vs. GLN-LPS

\section{$P M N \%$ and LDH in bronchoalveolar lavage fluid}

Inhaled LPS led to significant increases in PMN \% and LDH in all LPS-treated animals (NS-LPS and GLN-LPS) compared with the NS group ( $\mathrm{P}<0.0001$; Fig. 4 and Fig. 5). In the GLN-LPS group, pretreatment with GLN dramatically reduced PMN \% versus the NSLPS group receiving saline pretreatment $(49.5 \pm 12.3 \% v s$. $85.9 \pm 9.2 \%, \mathrm{P}<0.0001$; Fig. 4) and also decreased LDH 
activity $(79 \pm 14.4$ vs. $129.2 \pm 24.5 \mathrm{mAbs} / \mathrm{min}, \mathrm{P}<0.0001$; Fig. 5) in the BALF. Animals receiving GLN only did not show any increase in either PMN \% or LDH.

\section{A.}

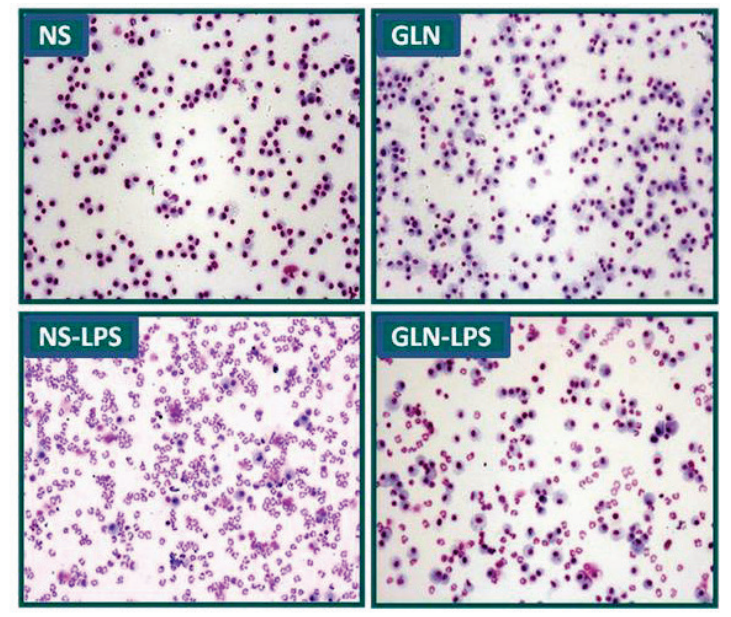

B.

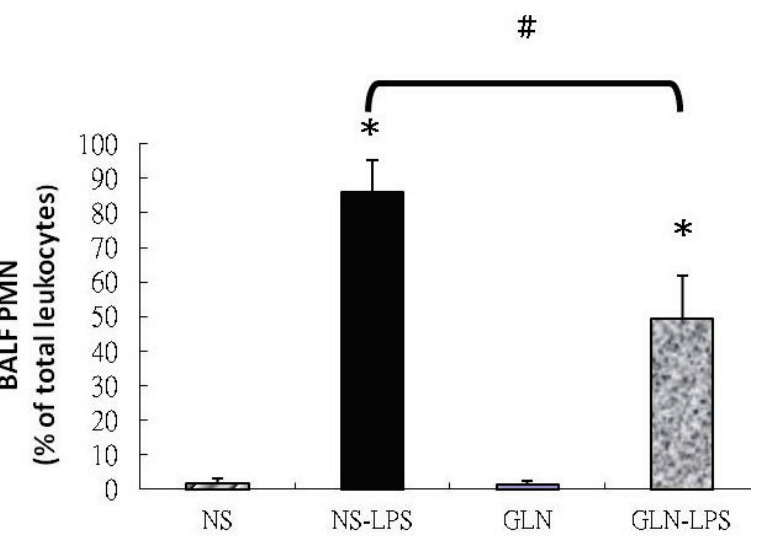

Fig. 4. A. Differential cell counts and images of BALF cells. PMNs were evaluated for air space migration by morphological analysis (original magnification, $\times 10$ ) The cells were stained with Geimsa and counted double blind under a microscope. B. PMN in BALF is presented as percent of total cells collected. LPS caused marked increases in these parameters. Pretreatment with GLN significantly attenuated the LPS effects. $* P<0.0001$ vs. NS, ${ }^{\#} \mathrm{P}<0.0001$ NS-LPS vs. GLN-LPS

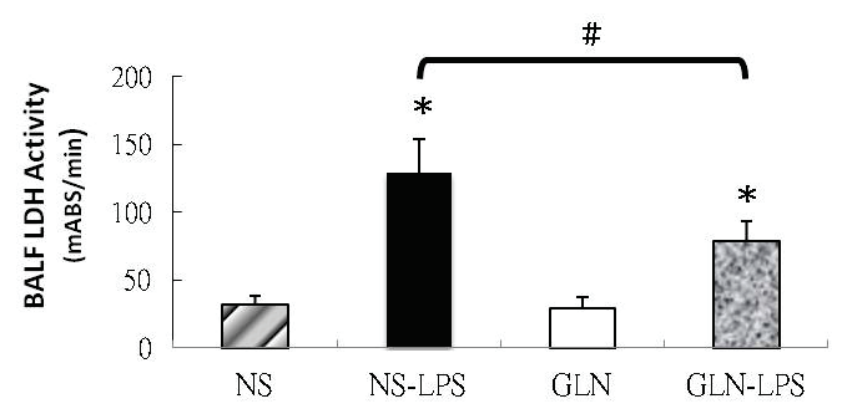

Fig. 5. Measurement of LDH activity in BALF. LPS caused marked increases in these parameters. Pretreatment with GLN significantly attenuated the LPS effects. $* \mathrm{P}<0.0001$ vs. NS, ${ }^{*} \mathrm{P}<0.0001$ NS-LPS vs. GLN-LPS

\section{Discussion}

In the present experiment, we used a rat model of acute lung injury induced by LPS inhalation. The degree of lung injury was assessed by various parameters including pathologic change in lung tissue, and LDH activity and PMN \% in BALF. The results imply that prophylactic inhalation of nebulized glutamine 1) enhanced lung tissue HSP72 synthesis, 2) decreased lung injury score, 3) reduced LDH activity and neutrophils in BALF. The beneficial effect of inhaled GLN is associated with its capacity to induce HSP72 expression in rats' lungs.

In previous animal and human studies, GLN has been administered as a continuous infusion, usually as a supplement to parenteral or enteral nutrition. Here, we report for the first time on GLN administered pharmacologically as a single dose via inhalation. The optimal dose of inhaled $1.5 \mathrm{~g} \mathrm{GLN}$ for these experiments is based on previous titration data (not shown) indicating the maximal HSP72 expression occurs at the lung tissue. Because GLN is given as a single dose over a short period, lung tissue levels of HSP72 may increase transiently; therefore we also examined multiple time points from 0 to $20 \mathrm{~h}$ after GLN inhalation and the 12-h time point was chosen to obtain the maximal response of HSP72 expression (Fig. 1).

In the experimental model of acute lung injury, the most wildly used method to quantify lung injury histologically is lung injury score. However, there is no agreement on which specific parameter should be adapted for scoring because the histological features varied with different models and different phases during acute lung injury (Matute-Bello et al. 2008, Koga et al. 2010). In our preliminary studies, we found that inhalation of nebulized LPS at a dose of $2 \mathrm{mg}$ was followed by an early phase response characterized by increases in BALF PMNs and LDH; and down-regulation of HSP72 at $8 \mathrm{~h}$ after LPS inhalation. The late phase response that followed was characterized by gradual normalization of BAL PMNs; and up-regulation of HSP72 at 16-32 $\mathrm{h}$ after LPS inhalation. It is noteworthy that during late phase response there was a hyaline membranes formation which was absent during early phase response. These findings indicate that certain parameters such as hyaline membrane formation for lung injury scoring were not uniform during early versus late phases of response. Accordingly, in our study we selected particular histological features such as edema and cell infiltration at 
time-point of 8-h which is regarded as most reliable during acute lung injury.

Studies have documented that significant neutrophil recruitment may be responsible for the production of inflammatory mediators after an LPS challenge. GLN supplementation markedly attenuates the increased bronchoalveolar epithelial permeability (Hulsewe et al. 2003, Tjader et al. 2007). Singleton and Wischmeyer (2007) also report that GLN has been shown to act against lung injury with the beneficial effect of healing lung parenchymal injuries and reduction of air leakage in rats. In this regard, GLN tends to play a protective role in alveolar barrier injury and inflammatory exudation. In connection with those studies, our results have also indicated that pretreatment by inhaled GLN could protect rats from LPS-induced ALI. Inhaled GLN markedly improved lung morphology, inhibited pulmonary edema formation and prevented infiltration of PMNs into alveoli (Fig. 3). The increases in BALF PMN counts and LDH activity induced by LPS were also abated by the prophylactic glutamine inhalation (Figs 4 and 5). It should be pointed out that the distribution of LDH isoenzymes varied in different tissues and the LDH3 is the predominate isoform in the lungs (Drent et al. 1996). Although we have not determined the isoform of $\mathrm{LDH}$, we have examined changes in total $\mathrm{LDH}$ in the circulating serum versus BALF. We found that total LDH in BALF was increased while serum $\mathrm{LDH}$ failed to change. These findings suggest that the LDH detected in BALF was most likely released locally from injured lung, and preassembly it consisted predominately the LDH-3 isoform.

Glutamine has a wide range of effects, including tissue protection, immune regulation, antioxidant capacity and stabilization of metabolism. Among these, enhanced HSP72 expression plays an important role in the antiinflammatory and tissue protection effects. The enhancement of HSP72 expression is not the only effect of GLN mediated protection but it is certainly the most significant and promising effect in the treatment of lung injury (Roth 2008, Singleton et al. 2005a,b, Wischmeyer et al. 2001, Vermeulen et al. 2007). The remarkable study done by Singleton and Wischmeyer (2007), utilizing mice with specific deletion of the HSP72 genes proved that GLN's mediated protection against lung injury is dependent on HSP72 expression. Another study done by Morrison et al. (2006) found that in heat shock factor-1 knockout cells, GLN's ability to generate an HSP response is lost and the concomitant protection of GLN's is also abolished. In agreement with much prior data, we deem that GLN mediated protection is highly correlated with HSP72 enhancement.

It is known that GLN is translocated out of lung and skeletal muscle in response to many forms of injury and stress (Tjader et al. 2007). Studies have indicated that in sepsis or lung injury, the down regulation of HSP72 expression may be due to a deficiency of lung tissue GLN. This relative decrease of HSP72 expression may lead to a worsening of lung tissue metabolism, subsequent lung injury, and organ failure (Bongers et al. 2007, Singleton et al. 2005a,b, Zhang et al. 2009, Jing et al. 2007). In the present study, we also observed that HSP72 expression appears to be down-regulated at 8-h after LPS inhalation (Fig. 2) and the down-regulation of HSP72 in turn aggravated lung injury; whereas prophylactic GLN inhalation not only prevented the occurrence of HSP72 down-regulation (Fig. 2), it also attenuated the LPS-induced lung injury. These observations suggest that the LPS associated HSP72 down-regulation could be abolished by GLN pretreatment, and in addition the protection effect of GLN is dependent on the level of HSP72 expression.

The limitation of this study is the lack of data investigating the effect of therapeutic GLN given at the time of LPS insult or thereafter, which would be of practical utility in a clinical setting. The present study does imply a positive outcome from prophylactic GLN inhalation, and an investigation of therapeutic applications of GLN for the treatment of acute lung injury is planned as part of our future studies.

Aerosol therapy is commonly used to administer certain drugs, and it provides a means to treat various pulmonary diseases. The advantage of aerosol therapy is the minimal systemic adverse effects with the use of smaller doses, besides giving a rapid response. In recent times, more and more new drugs, including bronchodilators, mucolytics, antibiotic, anti-inflammatory agents and insulin are becoming available for use as aerosol therapy (Rubin 2010). The increasing evidence, including the present study implicating GLN as a nontoxic, clinically relevant enhancer of HSP72 expression, supports the development of GLN for use as an inhalation agent providing a novel means of therapeutic intervention in a clinical setting.

In conclusion, the present results imply, for the first time that a single dose of prophylactic glutamine inhalation can enhance tissue HSP72 synthesis; these results also indicate that enhanced HSP72 may have 
functional significance as GLN-pretreated animals developed less lung damage following LPS inhalation. GLN inhalation appears to be a possible strategy to induce HSP72 to manage inflammatory airway diseases. Apart from these findings, future research could focus on the prophylactic function of inhaled GLN against occupational pneumoconiosis and other chronic lung diseases.

\section{Conflict of Interest}

There is no conflict of interest.

\section{Acknowledgements}

We thank Stuart Neff, MSTCM, for English writing assistance. This study was supported by grants from National Science Council (NSC100-2314-B-037-041) and Kaohsiung Medical University Hospital (KMUH1000M06).

\section{References}

BONGERS T, GRIFFITHS RD, MCARDLE A: Exogenous glutamine: the clinical evidence. Crit Care Med 35: S545S552, 2007.

BRUEMMER-SMITH S, STUBER F, SCHROEDER S: Protective functions of intracellular heat-shock protein (HSP) 70-expression in patients with severe sepsis. Intensive Care Med 27: 1835-1841, 2001.

CHEN HW, HSU C, LU TS, WANG SJ, YANG RC: Heat shock pretreatment prevents cardiac mitochondrial dysfunction during sepsis. Shock 20: 274-279, 2003.

CHUANG IC, LIU DD, KAO SJ, CHEN HI: N-acetylcysteine attenuates the acute lung injury caused by phorbol myristate acetate in isolated rat lungs. Pulm Pharmacol Ther 20: 726-733, 2007.

DE MAIO A: Heat shock proteins: facts, thoughts, and dreams. Shock 11: 1-12, 1999.

DONG HP, CHEN HW, HSU C, CHIU HY, LIN LC, YANG RC: Previous heat shock treatment attenuates lipopolysaccharide-induced hyporesponsiveness of platelets in rats. Shock 24: 239-244, 2005.

DRENT M, COBBEN NAM, HENDERSON RF, WOUTERS EFM, DIEIJEN-VISSER M: Usefulness of lactate dehydrogenase and its isoenzymes as indicators of lung damage or inflammation. Eur Respir 9: 1736-1742, 1996.

HOTCHKISS R, NUNNALLY I, LINDQUIST S, TAULIEN J, PERDRIZET G, KARL I: Hyperthermia protects mice against the lethal effects of endotoxin. Am J Physiol 265: R1447-R1457, 1993.

HUANG LJ, DONG HP, CHUANG IC, LIU MS, YANG RC: Attenuation of mitochondrial unfolded protein response is associated with hepatic dysfunction in septic rats shock. Shock 38: 642-648, 2012.

HULSEWE KW, VAN DER HULST RR, RAMSAY G, VAN BERLO CL, DEUTZ NE, SOETERS PB: Pulmonary glutamine production: effects of sepsis and pulmonary infiltrates. Intensive Care Med 29: 1833-1836, 2003.

JING L, WU Q, WANG F: Glutamine induces heat-shock protein and protects against Escherichia coli lipopolysaccharide-induced vascular hyporeactivity in rats. Crit Care 11: R34, 2007.

KARINCH AM, PAN M, LIN CM, STRANGE R, SOUBA WW: Glutamine metabolism in sepsis and infection. $J$ Nutr 131: 2535S-2538S, 2001.

KOGA H, HAGIWARA S, SHINGU C, MATSUMOTO S, YOKOI I, NOGUCHI T: Human atrial natriuretic peptide ameliorates LPS-induced acute lung injury in rats. Lung 188: 241-246, 2010.

MATUTE-BELlO G, FREVERT CW, MARTIN TR: Animal models of acute lung injury. Am J Physiol 295: L379L399, 2008.

MORRISON AL, DINGES M, SINGLETON KD, ODOMS K, WONG HR, WISCHMEYER PE: Glutamine's protection against cellular injury is dependent on heat shock factor-1. Am J Physiol 290: C1625-C1632, 2006.

ONG ES, GAO XP, XU N, PREDESCU D, RAHMAN A, BROMAN MT, JHO DH, MALIK AB: E. coli pneumonia induces CD18-independent airway neutrophil migration in the absence of increased lung vascular permeability. Am J Physiol 285: L879-L888, 2003.

RIBEIRO SP, VILLAR J, DOWNEY GP, EDELSON JD, SLUTSKY AS: Sodium arsenite induces heat shock protein72 kilodalton expression in the lungs and protects rats against sepsis. Crit Care Med 22: 922-929, 1994.

RITOSSA F: A new puffing pattern induced by temperature shock and DNP in Drosophila. Experientia 18: 571-573, 1962. 
ROTH E: Nonnutritive effects of glutamine. J Nutr 138: 2025S-2031S, 2008.

RUBIN BK: Air and soul: the science and application of aerosol therapy. Respir Care 55: 911-921, 2010.

SCHMITT E, GEHRMANN M, BRUNET M, MULTHOFF G, GARRIDO C: Intracellular and extracellular functions of heat shock proteins: repercussions in cancer therapy. J Leukoc Biol 81: 15-27, 2007.

SINGLETON KD, WISCHMEYER PE: Glutamine's protection against sepsis and lung injury is dependent on heat shock protein 70 expression. Am J Physiol 292: R1839-R1845, 2007.

SINGLETON KD, SERKOVA N, BECKEY VE, WISCHMEYER PE: Glutamine attenuates lung injury and improves survival after sepsis: role of enhanced heat shock protein expression. Crit Care Med 33: 1206-1213, 2005a.

SINGLETON KD, SERKOVA N, BANERJEE A, MENG X, GAMBONI-ROBERTSON F, WISCHMEYER PE: Glutamine attenuates endotoxine-induced lung metabolic dysfunction: potential role of enhanced heat shock protein 70. Nutrition 21: 214-223, 2005 b.

SUFIT A, WEITZEL LB, HAMIEL C, QUEENSLAND K, DAUBER I, ROOYACKERS O, WISCHMEYER PE: Pharmacologically dosed oral glutamine reduces myocardial injury in patients undergoing cardiac surgery: a randomized pilot feasibility trial. JPEN J Parenter Enteral Nutr 36: 556-561, 2012.

TJADER I, BERG A, WERNERMAN J: Exogenous glutamine - compensating a shortage? Crit Care Med 35: S553S556, 2007.

VERMEULEN MA, VAN DE POLL MC, LIGTHART-MELIS GC, DEJONG CH, VAN DEN TOL MP, BOELENS PG, VAN LEEUWEN PA: Specific amino acids in the critically ill patient - exogenous glutamine/arginine: a common denominator? Crit Care Med 35: S568-S576, 2007.

VILLAR J, RIBEIRO SP, MULLEN JB, KULISZEWSKI M, POST M, SLUTSKY AS: Induction of the heat shock response reduces mortality rate and organ damage in a sepsis-induced acute lung injury model. Crit Care Med 22: 914-921, 1994.

WANG SJ, CHEN HW, YANG RC: Pre-existent Hsp72 contributes to glutamine-induced hepatic hsp72 gene activation during heat shock recovery period in rat. Mol Nutr Food Res 56: 410-416, 2012.

WISCHMEYER PE: Glutamine: role in critical illness and ongoing clinical trials. Curr Opin Gastroenterol 24: 190$197,2008$.

WISCHMEYER PE, KAHANA M, WOLFSON R, REN H, MUSCH KM, CHANG EB: Glutamine induces heat shock protein and protects against endotoxin shock in the rat. $J$ Appl Physiol 90: 2403-2410, 2001.

ZHANG F, WANG X, PAN L, WANG W, LI N, LI J: Glutamine attenuated lipopolysaccharide-induced acute lung injury. Nutrition 25: 692-698, 2009. 